\title{
SIMILARITÉ ENTRE L'ALGÈBRE DE VOLTERRA ET UN QUOTIENT D'ALGÈBRE UNIFORME
}

\author{
par KONIN KOUA
}

(Received 3rd October 1989)

Two commutative Banach algebras $A$ and $B$ are said to be similar if there exists a Banach algebra $D$ such that $[x D]^{-}=D$ for some $x$ in $D$, and two one-to-one continuous homomorphisms $\phi: D \rightarrow A$ and $\psi: D \rightarrow B$ such that $\phi(D)$ is a dense ideal of $A$ and $\psi(D)$ a dense ideal of $B$.

We prove in this paper that the Volterra algebra $L_{*}^{1}(0,1)$ is similar to $A_{0} / e^{-z} A_{0}$ where $A_{0}$ is the commutative uniform, separable Banach algebra of all continuous functions on the closed right-hand half plane $\bar{H}$, analytic on $H$ and vanishing at infinity. We deduce from this result that multiplication by an element of $A_{0} / e^{-2} A_{0}$ is a compact mapping.

1980 Mathematics subject classification (1985 Revision): 46J10.

\section{Introduction}

Soient $A$ et $B$ deux algèbres de Banach commutatives. On dit que $A$ et $B$ sont similaires $([4, \mathrm{p} .116])$ s'il existe une algèbre de Banach commutative $D$ qui possède un idéal principal $a D$ dense et deux homomorphismes injectifs continus $\phi$ de $D$ dans $A$ et $\psi$ de $D$ dans $B$ tels que $\phi(D)$ soit un idéal dense dans $A$ et $\psi(D)$ soit un idéal dense dans B.

On remarque que cette définition implique que $\phi(a) A$ est dense dans $A$ et $\psi(a) B$ est dense dans $B$.

Si $A$ et $B$ sont similaires et si $\theta$ est un homorphisme continu de $A$ dans $B$, on dira que $\theta$ est un s-homomorphisme ([4, p. 123]) si le diagramme suivant est commutatif:

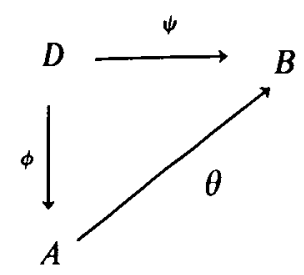

Soit $L^{1}\left(\mathbf{R}^{+}\right)$l'algèbre de Banach des (classes de) fonctions à valeurs complexes, Lebesgue intégrables sur $\mathbf{R}^{+}$et soit $\mathscr{I}$ l'idéal fermé de $L^{1}\left(\mathbf{R}^{+}\right)$formé des fonctions nulles presque partout sur $[0,1]$. L'algèbre quotient $L^{1}\left(\mathbf{R}^{+}\right) / \mathscr{I}$ est isomorphe à l'algèbre 
de Volterra $V=L_{*}^{1}(0,1)$ des (classes de) fonctions à valeurs complexes absolument intégrables sur $[0,1]$ (Remarque 2.2). On peut donc identifier $V$ à $L^{1}\left(\mathbf{R}^{+}\right) / \mathscr{I}$.

On se propose ici de montrer que $V$ est similaire à $A_{0} / I$, où $A_{0}$ est l'algèbre uniforme de toutes les fonctions continues sur le demi-plan droit fermé, analytiques dans le demiplan droit ouvert et tendant vers zéro à l'infini et où $I$ est l'idéal fermé de $A_{0}$ de la forme $e^{-z} A_{0}$.

La notion de similarité entre deux algèbres de Banach commutatives a été introduite par $J$. Esterle dans son étude du problème de l'existence d'une algèbre de Banach commutative topologiquement simple [4]. Le fait que deux algèbres de Banach commutatives à unité approchée bornée soient similaires implique d'une part l'existence d'une bijection entre l'ensemble de leurs idéaux fermés et implique d'autre part que les algèbres de leurs quasimultiplicateurs réguliers sont isomorphes ([4, p. 73 et p. 112]).

L"idée que les algèbres $L^{\mathbf{1}}\left(\mathbf{R}^{+}\right) / \mathscr{I}$ et $A_{0} / I$ pouvaient être similaires nous a été suggérée par le travail de E. Strouse sur les idéaux fermés de l'algèbre de Volterra en plusieurs variables [8].

Notre résultat de similarité permet de retrouver en une variable le résultat de [8]; mais ceci n'apporte rien de nouveau car la structure des idéaux fermés de l'algèbre de Volterra est banale et bien connue (voir per exemple [2, Théorème 7-9]). Par contre, ce résultat permet de ramener l'étude des quasimultiplicateurs réguliers de $V$ à ceux de $A_{0} / I$, ce qui permet d'espérer obtenir ultérieurement une caractérisation concrète des quasimultiplicateurs de $V$. Ce point ne sera pas développé dans le présent article.

Pour finir, nous établissons le fait que si deux algèbres de Banach sont similaires et si la multiplication par tout élément est compacte dans l'une, il en est de même de la multiplication par tout élément dans l'autre (Proposition 4.1). On en déduit que la multiplication dans $A_{0} / I$ est compacte.

Je remercie E. Strouse de m'avoir communiqué son manuscrit et je remercie également Jean Esterle pour ses fructueux conseils pendant la réalisation de ce travail.

\section{Préliminaires}

Soit $H$ le demi-plan droit ouvert $H=\{z \in \mathbf{C} / \operatorname{Re} z>0\}$. Soit $A_{0}$ l'algèbre de toutes les fonctions $f$ continues sur $\vec{H}$, analytiques dans $H$ et vérifiant

$$
\lim _{\substack{\operatorname{Re} z \geqq 0 \\|z| \rightarrow+\infty}}|f(z)|=0 .
$$

Pour chaque $f$ de $A_{0}$, on pose $\|f\|_{\infty}=\sup _{\mathrm{Rez} \geq 0}|f(z)|$. D'après le principe de PhragménLindelöf $\left(\left[1\right.\right.$, p. 3]) on a $\|f\|_{\infty}=\sup _{y \in \mathbf{R}}|f(i y)| ; f \in A_{0}$. Munie de cette norme, $A_{0}$ est une algèbre de Banach commutative, uniforme et séparable.

Proposition 2.1. L'élément $\beta$ de $A_{0}$, défini par

$$
\beta(z)=\frac{1}{(1+z)^{2}}
$$


vérifie $\left[\beta A_{0}\right]^{-}=A_{0}$.

Preuve. Soit

$$
\tau: z \mapsto \frac{z-1}{z+1}
$$

la transformation conforme du demi-plan droit $H$ sur le disque unité $D=\{z \in C /|z|<1\}$, soit $A(D)$ l'algèbre du disque et soit

$$
M_{1}=\{f \in A(D) \mid f(1)=0\}
$$

Soit $\mu$ l'application:

$$
z \mapsto \frac{1-z}{2}
$$

L'application $\rho: f \mapsto f \circ \tau$ est un isomorphisme isométrique de $M_{1}$ sur $A_{0}$. On a $\beta=(\rho(\mu))^{2}$. Comme $\left[\mu M_{1}\right]^{-}=M_{1}$, puisque les polynômes en $\mu$ sans terme constant sont denses dans $M_{1}$, on a $\left[\rho(\mu) A_{0}\right]^{-}=A_{0}$, et donc $\left[\beta A_{0}\right]^{-}=A_{0}$.

Soint maintenant la fonction $F: z \mapsto e^{-z} ; \operatorname{Re} z>0$. Alors la multiplication par $F$ est un multiplicateur sur $A_{0}$ et $\|F g\|_{\infty}=\|g\|_{\infty}$ pour tout élément $g$ de $A_{0}$.

Donc l'idéal $I$ de la forme $F A_{0}$, noté $e^{-z} A_{0}$, est un idéal propre fermé de $A_{0}$. Un caractère $\chi$ de $A_{0}$ est de la forme $\chi(f)=f(t) ; t \in \bar{H}$. Puisque $F(t) \neq 0$ pour tout $t \in \bar{H}$, aucun caractère sur $A_{0}$ ne s'annule sur $I$. Donc l'algèbre quotient $A_{0} / I$ est une algèbre de Banach radicale ([7, p. 87]).

Soit $L^{1}\left(\mathbf{R}^{+}\right)$l'espace des fonctions $f$ définies sur $\mathbf{R}^{+}$, à valeurs complexes et Lebesgue intégrables. Muni de la norme $\|f\|_{1}=\int_{0}^{\infty}|f(t)| d t$ et du produit de convolution (défini presque partout):

$$
(f * g)(t)=\int_{0}^{t} f(t-s) g(s) d s, f, g \in L^{1}\left(\mathbf{R}^{+}\right),
$$

$L^{1}\left(\mathbf{R}^{+}\right)$est une algèbre de Banach commutative, semi-simple sans unité $([6, \mathrm{p} .94])$.

L'ensemble $L^{1}\left(\mathbf{R}^{+}\right)$de tous les caractères sur $L^{1}\left(\mathbf{R}^{+}\right)$peut être identifié à $\bar{H}$ par l'application: $\lambda \mapsto \phi_{\lambda}$ de $\bar{H}$ dans $\left.L^{\widehat{T}} \widehat{\mathbf{R}}^{+}\right)$où $\phi_{\lambda}$ est l'application:

$$
f \mapsto \int_{0}^{+\infty} f(t) e^{-\lambda t} d t=\mathscr{L}(f)(\lambda) ; f \in L^{1}\left(\mathbf{R}^{+}\right)
$$

La transformée de Gelfand est donc précisément la transformée de Laplace $\mathscr{L}$ : 
$f \mapsto \mathscr{L}(f)$. Il est bien connu que $\mathscr{L}$ est un homomorphisme injectif continu de $L^{1}\left(\mathbf{R}^{+}\right)$ dans (et non sur) $A_{0}$.

Soit $\mathscr{I}$ l'ideal fermé de $L^{1}\left(\mathbf{R}^{+}\right)$formé des fonctions nulles presque partout sur $[0,1]$. On note $V=L_{*}^{1}(0,1)$ l'algèbre de Volterra, c'est-à-dire l'algèbre de Banach des fonctions $f$ à valeurs complexes, absolument intégrables sur $[0,1]$, muni de la norme $\|f\|_{1}=$ $\int_{0}^{1}|f(t)| d t$ et du produit de convolution (défini presque partout):

$$
(f * g)(t)=\int_{0}^{t} f(t-s) g(s) d s ; 0 \leqq t \leqq 1 .
$$

Remarque 2.2. $\quad V$ est isomorphe à $L^{1}\left(\mathbf{R}^{+}\right) / \mathscr{I}$.

Preuve. Soit $R: f \mapsto f_{1[0,1]}$ l'application restriction de $L^{1}\left(\mathbf{R}^{+}\right)$dans $V$. C'est un homomorphisme surjectif. Puisque $\operatorname{ker} R=\mathscr{I}$, on a que $L^{1}\left(\mathbf{R}^{+}\right) / \mathscr{I}$ est isomorphe à $V$.

Soit $L^{\infty}\left(\mathbf{R}^{+}\right)$l'espace de Banach des fonctions $f$ à valeurs complexes essentiellement bornées sur $\mathbf{R}^{+}$, muni de la norme:

$$
\|f\|_{\infty}=\underset{t \geqq 0}{\operatorname{ess} \sup }|f(t)| .
$$

On peut définir la transformée de Laplace sur $L^{\infty}\left(\mathbf{R}^{+}\right)$par la formule:

$$
\mathscr{L}(f)(z)=\int_{0}^{+\infty} f(t) e^{-z t} d t
$$

Dans ce cas, $\mathscr{L}(f)$ est seulement définie pour $\operatorname{Re} z>0$, et elle n'est pas en général bornée sur $H$.

On prolonge implicitement à $\mathbf{R}$ les fonctions définies sur un sous-ensemble de $\mathbf{R}^{+}$en les supposant nulles sur le complémentaire de leur domaine. On peut considérer $V$ comme un sous-espace de $L^{1}\left(\mathbf{R}^{+}\right)$.

Désormais on notera $V_{1}=L^{1}\left(\mathbf{R}^{+}\right) / \mathscr{I}, W=A_{0} / I, \theta: A_{0} \mapsto W$ et $\pi: L^{1}\left(\mathbf{R}^{+}\right) \mapsto V_{1}$ les surjections canoniques. $V_{1}$ est identifiable à $V$ (Remarque 2.2); l'application

$$
R: f \mapsto f_{1[0,1]}
$$

donne une extension de $\pi$ à $L^{\infty}\left(\mathbf{R}^{+}\right)$que nous noterons également $\pi$.

Remarque 2.3. $\mathscr{L}$ étant la transformée de Laplace qui applique $L^{1}\left(\mathbf{R}^{+}\right)$dans $A_{0}$, on a

$$
\operatorname{ker} \theta \circ \mathscr{L}=\mathscr{I} \text {. }
$$

Preuve. Ceci résulte de ce que $\mathscr{I}=\mathscr{L}^{-1}(I)$, c'est-à-dire $f \in \mathscr{I}$ si et seulement si $\mathscr{L}(f) \in I([2$, preuve du théorème 7-4]). 
Remarque 2.4. Il existe un homomorphisme injectif continu de $V_{1}$ dans $W$ noté $\tilde{\mathscr{L}}$ et tel que $\theta \circ \mathscr{L}=\tilde{\mathscr{L}}$ ॰ $\pi$.

Preuve. Ceci résulte de la Remarque 2.3 et de la factorisation canonique des applications.

Lemma 2.5. ([8, Lemme 4-4]). Si $f \in L^{\infty}\left(\mathbf{R}^{+}\right)$est telle que $\mathscr{L}(f) \in A_{0}$ et si $f(t)=0$ pour presque tout $t \in[0,1]$, alors $\mathscr{L}(f) \in I$.

Proposition 2.6. Si $f \in L^{\infty}\left(\mathbf{R}^{+}\right)$et si $\mathscr{L}(f) \in A_{0}$, alors $(\tilde{\mathscr{L}} \circ \pi)(f)=(\theta \circ \mathscr{L})(f)$.

Preuve. Soit $f \in L^{\infty}\left(\mathbf{R}^{+}\right)$. Posons $f_{1}=\pi(f)$ et $f_{2}=f-f_{1}$. Alors $f_{1} \in L^{1}\left(\mathbf{R}^{+}\right)$et $\mathscr{L}\left(f_{1}\right) \in A_{0}$. On en déduit que $\mathscr{L}\left(f_{2}\right) \in A_{0}$ car $\mathscr{L}(f) \in A_{0} . f_{2}$ vérifie donc les hypothèses du Lemme 2.5; donc $\mathscr{L}\left(f_{2}\right) \in I$, Il s'ensuit que $(\theta \circ \mathscr{L})\left(f_{2}\right)=0$. Donc

$$
(\theta \circ \mathscr{L})(f)=(\theta \circ \mathscr{L})\left(f_{1}\right)=(\tilde{\mathscr{L}} \circ \pi)\left(f_{1}\right)=\left(\tilde{\mathscr{L}}\left(\pi\left(f_{1}\right)\right)=(\tilde{\mathscr{L}} \pi)(f)\right.
$$

\section{Similarité entre $V$ et $W$}

On pose $\gamma=\theta(\beta)$ où $\beta$ est l'élément de $A_{0}$ défini à la Proposition 2.1. On considère l'ensemble $\mathscr{D}=\gamma W$.

Remarque 3.1. $\mathscr{D}$ est dense dans $W$.

En effet pour tout $y \in W$, il existe $x \in A_{0}$ tel que $y=\theta(x)$, donc il existe une suite $\left(x_{n}\right)_{n}$ d'éléments de $A_{0}$ telle que $y=\theta\left(\lim _{n} \beta x_{n}\right)$ d'après la Proposition 2.1.

En posant $d_{n}=\gamma \theta\left(x_{n}\right)$ pour chaque $n$, on a $y=\lim _{n} d_{n}$, d'où le résultat.

Remarque 3.2. $\gamma$ n'est pas un diviseur de zéro dans $W$.

En effet si $\gamma \cdot u=0$, alors $u=0$, car $[\gamma W]^{-}=W$ d'après la Remarque 3.1 et $W$ a une unité approchée bornée ([7, p. 87]).

Remarque 3.3. L'application $q$ définie pour $d=\gamma u$ par $q(d)=\|u\|_{W}$ est une norme d'algebre sur $\mathscr{D}$.

Preuve. Il suffit de vérifier l'inégalité du produit.

Pour $d=\gamma u$ et $d^{\prime}=\gamma u^{\prime}$ on a

$$
q\left(d d^{\prime}\right)=q\left(\gamma^{2} u u^{\prime}\right)=\left\|\gamma u u^{\prime}\right\| \leqq\|\gamma\|\|u\|\left\|u^{\prime}\right\| \leqq\|u\|\left\|u^{\prime}\right\|
$$

$\operatorname{car}\|\gamma\| \leqq 1 ;$ donc $q\left(d d^{\prime}\right) \leqq q(d) q\left(d^{\prime}\right) ; d, d^{\prime} \in \mathscr{D}$. 
Proposition 3.4. Muni de laddition et de la multiplication définies sur $W$ et de la norme $q, \mathscr{D}$ est une algèvre de Banach et l'élément $\gamma^{2}$ de $\mathscr{D}$ vérifie $\left[\gamma^{2} \mathscr{D}\right]^{-}=\mathscr{D}$.

Preuve. L'application: $d \mapsto(d / \gamma)$ est une isométrie de $\mathscr{D}$ sur $W$; on en déduit que $\mathscr{D}$ est une algèbre de Banach. On a $\left[\gamma^{2} W\right]^{-}=W$ car $[\gamma W]^{-}=W$ (Remarque 3.1).

Soit $d=\gamma u$ un élément de $\mathscr{D}$. Il existe une suite $\left(u_{n}\right)_{n \geqq 1}$ d'éléments de $W$ telle que $\left\|u-\gamma^{2} u_{n}\right\|_{w} \rightarrow 0$ quand $n \rightarrow+\infty$. Or $\left\|u-\gamma^{2} u_{n}\right\|_{w}=q\left(\gamma u-\gamma^{3} u_{n}\right)=q\left(\gamma u-\gamma^{2}\left(\gamma u_{n}\right)\right)$. Donc en posant $d_{n}=\gamma u_{n}$ pour chaque $n$, on obtient une suite $\left(d_{n}\right)_{n \geqq 1}$ de $\mathscr{D}$ telle que $q\left(d-\gamma^{2} d_{n}\right) \rightarrow 0$ quand $n \mapsto+\infty$. Donc l'élément $\gamma^{2}$ de $\mathscr{D}$ vérifie $\left[\gamma^{2} \mathscr{D}\right]^{-}=\mathscr{D}$.

Si $g$ est un élément de $A_{0}$ intégrable sur l'axe imaginaire, alors on peut définir $\mathscr{L}^{-1}(g)(t)$ pour $t \in[0,+\infty[$ par la formule:

$$
\mathscr{L}^{-1}(g)(t)=\frac{1}{2 \pi} \int_{-\infty}^{+\infty} g(i y) e^{i y t} d y
$$

Le lemme suivant est standard:

Lemme 3.5. (i) Sif $\in A_{0}$, alors $\left\|\mathscr{L}^{-1}(\beta f)\right\|_{\infty} \leqq \frac{1}{2}\|f\|_{\infty}$, avec $\left\|\mathscr{L}^{-1}(\beta f)\right\|_{\infty}=$ $\sup _{t \geqq 0}\left|\mathscr{L}^{-1}(\beta f)(t)\right|$. (ii) Si $f \in I$, alors $\mathscr{L}^{-1}(\beta f)=0$ sur $[0,1]$.

Preuve. (i) Est évident. (ii) Si $f \in I$, alors il existe $h \in A_{0}$ telle que $f(z)=e^{-z} h(z)$.

$$
\mathscr{L}^{-1}(\beta f)(t)=\frac{1}{2 \pi} \int_{-\infty}^{+\infty}(\beta f)(i y) e^{i y t} d y=\frac{1}{2 \pi} \int_{-\infty}^{+\infty}(\beta h)(i y) e^{i y(t-1)} d y=\mathscr{L}^{-1}(\beta h)(t-1) .
$$

Or pour tout $l \in A_{0}, \mathscr{L}^{-1}(\beta I)(t)$ existe pour tout $t \in \mathbf{R}$ et est nul pour tout $t$ négatif.

Donc $\mathscr{L}^{-1}(\beta f)(t)=\mathscr{L}^{-1}(\beta h)(t-1)=0$ si $t \leqq 1$. D'où l'on déduit que $\mathscr{L}^{-1}(\beta f)$ s'annule sur $[0,1]$.

Soit l'application $\phi$ de $\mathscr{D}$ dans $V_{1}$ définie pour tout élément $d=\gamma u$ de $\mathscr{D}$ par la formule $\phi(d)=\pi\left(\mathscr{L}^{-1}(\beta f)\right)$ où $f \in A_{0}$ est tel que $u=\theta(f)$ c'est-à-dire $\beta f \in \theta^{-1}(d)$.

$\phi$ est bien définie car l'écriture $d=\gamma u$ avec $u \in W$ est unique pour $d \in \mathscr{D}$ et si $\theta(f)=\theta(g)=u$ avec $f$ et $g$ dans $A_{0}$, on a $f-g \in I$. Il s'ensuit que $\mathscr{L}^{-1}(\beta(f-g))=0$ sur $[0,1]$ d'après le Lemme 3.5 (ii). Donc $\pi\left(\mathscr{L}^{-1}(\beta f)\right)=\pi\left(\mathscr{L}^{-1}(\beta g)\right)$.

Proposition 3.6. $\phi$ est un homomorphisme injectif continu, et $(\tilde{\mathscr{L}} \circ \phi)(f)=f ;(f \in \mathscr{D})$.

Preuve. Il est ëvident que $\phi$ est un homomorphisme.

Soit $a=\theta(\beta f) ; f \in A_{0}$, un élément de $\mathscr{D}$. On a $(\tilde{\mathscr{L}} \circ \phi)(a)=\mathscr{L}\left(\left(\pi \circ \mathscr{L}^{-1}\right)(\beta f)\right)$. Mais $\mathscr{L}^{-1}(\beta f) \in L^{\infty}\left(\mathbf{R}^{+}\right)$et $\mathscr{L}\left(\mathscr{L}^{-1}(\beta f)\right)=\beta f \in A_{0}$. Il résulte alors de la Proposition 2.6 que $(\tilde{\mathscr{L}} \circ \pi)\left(\mathscr{L}^{-1}(\beta f)\right)=(\theta \circ \mathscr{L})\left(\mathscr{L}^{-1}(\beta f)\right)=\theta(\beta f)=a$. Autrement dit $\mathscr{L} \circ \phi=I d_{g}$ et nous en déduisons en particulier que $\phi$ est injectif.

Montrons que $\phi$ est continu: soit $\left(d_{n}=\gamma u_{n}\right)_{n \geq 1}$ une suite d'éléments de $\mathscr{D}$ telle que $q\left(d_{n}\right) \rightarrow 0$ quand $n \rightarrow+\infty$. On a $\phi\left(d_{n}\right)=\pi\left(\mathscr{L}^{-1}\left(\beta f_{n}\right)\right)$ où l'on choisit la suite $\left(f_{n}\right)_{n \geqq 1}$ de $A_{0}$ 
telle que $\left\|f_{n}\right\|_{\infty} \leqq\left\|u_{n}\right\|_{W}+(1 / n)$ pour tout $n$. On a $\left\|\mathscr{L}^{-1}\left(\beta f_{n}\right)\right\|_{\infty} \leqq \frac{1}{2}\left\|f_{n}\right\|_{\infty}$ (Lemme 3.5(i)). Donc

$$
\left\|\phi\left(d_{n}\right)\right\|=\left\|\pi\left(\mathscr{L}^{-1}\left(\beta f_{n}\right)\right)\right\| \leqq\left\|\mathscr{L}^{-1}\left(\beta f_{n}\right)\right\|_{\infty} \leqq \frac{1}{2}\left\|f_{n}\right\|_{\infty} \leqq \frac{1}{2}\left(\left\|u_{n}\right\|_{W}+1 / n\right) .
$$

Donc $\phi\left(d_{n}\right) \rightarrow 0$ quand $n \rightarrow+\infty$ et $\phi$ est continu.

Lemme 3.7. Soit $\alpha$ Pélément de $L^{1}\left(\mathbf{R}^{+}\right)$défini par $\alpha(x)=x e^{-x}$. Alors $\pi(\alpha) \cdot V_{1}$ est contenu dans $\phi(\mathscr{D})$.

Preuve. Pour tout élément $f$ de $V_{1}$, on a

$$
\begin{aligned}
\tilde{\mathscr{L}}(\pi(\alpha) * f) & =\tilde{\mathscr{L}}(\pi(\alpha)) \cdot \tilde{\mathscr{L}}(f) \\
& =(\theta \circ \mathscr{L})(\alpha) \cdot \tilde{\mathscr{L}}(f) \\
& =\theta(\beta) \cdot \tilde{\mathscr{L}}(f) \quad \operatorname{car} \beta=\mathscr{L}(\alpha) \\
& =\gamma \cdot \tilde{\mathscr{L}}(f) .
\end{aligned}
$$

Ceci montre que $\tilde{\mathscr{L}}(\pi(\alpha) * f)$ est dans $\mathscr{D}$ et comme $\tilde{\mathscr{L}} \circ \phi=I d_{\mathscr{Q}}$ on a $\tilde{\mathscr{L}}(\pi(\alpha) * f)=$ $(\tilde{\mathscr{L}} \circ \phi)(\tilde{\mathscr{L}}(\pi(\alpha) * f))=\tilde{\mathscr{L}}(\phi(\tilde{\mathscr{L}}(\pi(\alpha) * f)))$. Donc $\pi(\alpha) * f=\phi(\tilde{\mathscr{L}}(\pi(\alpha) * f))$ puisque $\tilde{\mathscr{L}}$ est injectif. Ainsi $\pi(\alpha) * f \in \phi(\mathscr{D})$ pour tout $f$ de $V_{1}$, d'où l'inclusion $\pi(\alpha) \cdot V_{1} \subset \phi(\mathscr{D})$.

Nous sommes maintenant en mesure d'établir le résultat principal de cet article:

Théorème 3.8. L'algèbre de Volterra $V=L_{*}^{1}(0,1)$ est similaire à Talgèbre de Banach commutative, radicale à unité approchée bornée $A_{0} / I=W$, et l'application $\tilde{\mathscr{L}}$ de $V$ dans $W$ est un s-homomorphisme.

Preuve. Grâce au fait que $\tilde{\mathscr{L}} \circ \phi=I d_{\mathscr{D}}$ et grâce aux Remarques 2.2 et 3.1 et aux Propositions 3.4 et 3.6 , il suffit de montrer que $\phi(\mathscr{D})$ est un idéal dense de $V_{1}$ pour établir le théorème.

Soient donc $f \in \phi(\mathscr{D}), g \in V_{1}$ et soit $d$ l'élément de $\mathscr{D}$ tel que $f=\phi(d)$. Posons $d^{\prime}=\tilde{\mathscr{L}}(g)$. Alors $d d^{\prime} \in \mathscr{D}$ puisque $\mathscr{D}$ est un idéal de $W$. Comme $\tilde{\mathscr{L}} \circ \phi=I d_{\mathscr{g}}$, on a:

$$
\begin{aligned}
\tilde{\mathscr{L}}\left(\phi\left(d d^{\prime}\right)\right)=d d^{\prime}=((\tilde{\mathscr{L}} \circ \phi)(d)) \cdot d^{\prime} & \\
& =\tilde{\mathscr{L}}(\phi(d)) \cdot \tilde{\mathscr{L}}(g) \\
& =\tilde{\mathscr{L}}(\phi(d) * g) .
\end{aligned}
$$

L'injectivité de $\tilde{\mathscr{L}}$ entraine qui $\phi\left(d d^{\prime}\right)=\phi(d) * g=f * g$, ce qui implique que $f * g \in \phi(\mathscr{D})$ pour tout $f$ dans $\phi(\mathscr{D})$ et tout $g$ dans $V_{1}$. Donc $\phi(\mathscr{D})$ est bien un idéal de $V_{1}$. $\alpha$ étant 
l'application définie au Lemme 3.7, on sait que $\left[\alpha * L^{1}\left(\mathbf{R}^{+}\right)\right]^{-}=L^{1}\left(\mathbf{R}^{+}\right)$(voir par exemple [3, p. 47]). Donc $\pi(\alpha) V_{1}$ est dense dans $V_{1}$ et par suite $\phi(\mathscr{D})$ est dense puisque $\pi(\alpha) V_{1}$ est contenu dans $\phi(\mathscr{D})$ d'après le Lemme 3.7 , ce qui achève la démonstration.

\section{Similarité et compacité de la multiplication par un element}

Proposition 4.1. Soient $A$ et $B$ deux algèbres de Banach commutatives similaires. Si la multiplication par tout élément de A est compacte, alors la multiplication par tout élément de B lest aussi.

Preuve. $A$ et $B$ étant similaires, il existe une algèbre de Banach commutative $D$ qui possede un idéal principal $a D$ dense et deux homomorphismes injectifs continus $\phi$ de $D$ dans $A$ et $\psi$ de $D$ dans $B$ tels que $\phi(D)$ est un idéal dense de $A$ et $\psi(D)$ est un idéal dense de $B$.

Soit $\left(d_{n}\right)_{n \geqq 1}$ une suite bornée d'éléments de $D$. La suite $\left(\phi\left(d_{n}\right)\right)_{n \geqq 1}$ est alors bornée dans $A$. Il existe donc une suite extraite $\left(\phi\left(d_{n_{i}}\right)\right)_{i \geqq 1}$ telle que $\left(\phi\left(a d_{n_{i}}\right)\right)_{i \geqq 1}$ converge dans $A$ puisque la multiplication est compacte dans $A$.

Il existe une constant $k>0$ telle que $\left\|\phi^{-1}\left(\phi(d) a^{\prime}\right)\right\|_{D} \leqq k\|d\|_{D}\left\|a^{\prime}\right\|_{A}$ pour tout $d$ dans $D$ et tout $a^{\prime}$ dans $A$ ([4, Lemme 7-1]). En particulier $\left\|d_{1} d_{2}\right\|_{D} \leqq k\left\|d_{1}\right\|_{D}\left\|\phi\left(d_{2}\right)\right\|_{A} ; d_{1}$ et $d_{2}$ appartenant à $D$. Donc pour tout élément $d$ de $D$, on a:

$$
\left\|d a d_{n_{i}}-d a d_{n}\right\|_{D} \leqq k\|d\|_{D}\left\|\phi\left(a d_{n_{i}}\right)-\phi\left(a d_{n j}\right)\right\|_{A} .
$$

On en déduit que $\left(a d d_{n_{i}}\right)_{i \geq 1}$ converge dans $D$. Donc la multiplication par $d$ est compacte pour tout $d \in a D$ et par conséquent pour tout $d \in D$.

Soit maintenant $\left(b_{n}\right)_{n \geqq 1}$ une suite bornée d'éléments de $B$. Alors la suite $\left(\psi^{-1}\left(\psi(a) b_{n}\right)\right)_{n \geqq 1}$ est bornée dans $D$ car il existe une constante $C>0$ telle que:

$$
\left\|\psi^{-1}(\psi(d) b)\right\|_{D} \leqq C\|d\|_{D}\|b\|_{B} \text { pour } d \in D, b \in B .
$$

Donc pour tout $d$ de $D$, il existe une sous-suite $\left(b_{n_{1}}\right)_{i \geqq 1}$ telle que la suite $\left(d . \psi^{-1}\left(\psi(a) b_{n_{i}}\right)\right)_{i \geqq 1}$ cnverge dans $D$. On en déduit que:

$$
\left(\psi(d) . \psi(a) b_{n_{i}}\right)_{i \geqq 1}=\left(\psi(a d) \cdot b_{n_{i}}\right)_{i \geqq 1}
$$

converge dans $B$. Ainsi la multiplication par $\psi(d a)$ est compacte dans $B$ pour tout $d$ de $D$. Comme $\psi(a D)$ est dense dans $B$, la multiplication par tout élément de $B$ est compacte, ce qui achève la démonstration.

Corollaire 4.2. La multiplication par tout élément de $A_{0} / I$ est compacte.

Preuve. Il est bien connu que la multiplication dans l'algèbre de Volterra $V$ est compacte et $V$ et $A_{0} / I$ sont similaires (Théorème 3.8). 
Remarque 4.3. L'image $L$ de $I$ par lisomorphisme entre $A_{0}$ et $M_{i}$ introduit à la Proposition 2.1 est un idéal de l'algèbre du disque $A(D)$ tel que

$$
Z(L)=\{z \in \overline{\mathscr{D}} / f(z)=0 ; f \in L\}
$$

soit réduit à $\{1\}$. Le Corollaire 4.2 est donc un cas particulier de [5, Corollaire 2.14].

\section{RÉFÉRENCES}

1. R. Boas, Entire functions, Pure and Appl. Math. 5 (Academic Press Inc., New York, 1954).

2. H. G. Dales, Automatic continuity: a survey, Bull. London Math. Soc. 10 (1978), 129-183.

3. J. ESTERLE, Elements for a classification of commutative radical Banach algebras, (Lecture Notes in Math. 975, Springer-Verlag, Berlin, Heidelberg, New York, 1983), 4-65.

4. J. Esterle, Quasimultipliers, representations of $H^{\infty}$ and the closed ideal problem for commutative Banach algebras, (Lecture Notes in Math. 975, Springer-Verlag, Berlin, Heidelberg, New York, 1983), 66-162.

5. J. Esterle, E. Strouse and F. Zouakia, Theorems of Katznelson-Tzafrini type, J. Funct. Anal. 94 (1990), 273-287.

6. I. M. Gelfand, D. A. Raikov et G. E. Chilov, Les anneaux normés commutatifs (Monographies internatioanles de mathématiques modernes, Gauthier Villars, Paris, 1964).

7. A. M. Sinclair, Continuous semi-groups in Banach algebras (London Math. Soc. Lec. Notes 63, 1982).

8. E. Strouse, Closed ideals in convolutions algebras and the Laplace transform, Michigan Math. J. 35 (1988), 185-196.

MATHEMATIQUeS ET INFORMATIQUe

UNIVERSITÉ BORDEAUX 1

351 Cours de la Liberation

33405 Talence Cedex

FRANCE 